\title{
Influence of CVD diamond growth conditions and misorientation angle on nitrogen incorporation
}

\author{
M.A. Lobaev ${ }^{1}$, A.M. Gorbachev ${ }^{1}$, S.A. Bogdanov ${ }^{1}$, A.L.Vikharev ${ }^{1}$, D.B. Radishev ${ }^{1}$, \\ V.A. Isaev ${ }^{1}$, V.V. Chernov ${ }^{1}$, M.N. Drozdov ${ }^{1}$, P.A. Yunin ${ }^{1}$ \\ ${ }^{1}$ Federal Research Center The Institute of Applied Physics of the Russian Academy of Sciences, \\ Nizhny Novgorod, Russia, bogser@appl.sci-nnov.ru
}

CVD diamonds with NV-centers (nitrogen vacancy centers) are now considered as the most promising solidstate media for the realization of component base for quantum information processing due to the unique properties of NV-center electronic transitions, which allow initialization and read-out of it's spin state [1].

The concentration and spin properties of NV-centers depend on nitrogen concentration and therefore it is important to understand the dependence of nitrogen incorporation in delta-layers on CVD growth conditions. In this work, delta-layers (1-5 nm thick) doped with nitrogen were obtained during CVD synthesis using the unique setup for delta doping created in IAP RAS [2]. It was shown that the subsequent CVD growth with and without nitrogen addition allows obtaining a two-dimensional structure of the NV centers with a given concentration and any required depth that may be determined with an accuracy of up to 1-2 nanometers. Also the influence of substrate temperature, nitrogen flow and methane content on nitrogen incorporation was investigated by the growth of nitrogen doped layers on (100) and (111)-oriented samples.

The method of delta doping in contrast to the method of ion implantation does not produce lattice defects and allows controlling the depth of the NV-center to within a few nanometers. CVD growth allows producing singlecrystal diamond of high crystalline perfection with a low content of impurities that does not vary from sample to sample. Due to the absence of lattice damage, as in the case of ion implantation, NV-centers produced by deltadoping will have better properties (e.g. higher spin coherence times).

CVD diamond growth of nitrogen-doped layers was performed in the novel CVD reactor. The main features of the reactor are: 1) rapid gas switching; 2) laminar gas flow; 3) axial symmetric resonant mode - symmetric discharge; 4) slow growth of diamond (40-100 nm/h without nitrogen addition). We achieve rapid gas switching from one input gas to another by a home-made electronic switch. The residence time of our reactor is approximately $5 \mathrm{~s}$. The reactor is also capable for the growth of delta-layers doped with any other impurities, such as nitrogen.

It is well-known, that incorporation of impurities during CVD diamond growth is highly dependent on the substrate orientation, and could also be affected by the misorientation angle. In order to eliminate the influence of substrate, each of the experiment was performed on one sample, varying growth conditions in a one growth process, resulting in the growth of multiple thin nitrogendoped layers (see Fig. 1). Moreover, in this work we investigated the dependence of nitrogen incorporation effi- ciency on the misorientation angle using the (100)oriented sample with multiple facets polished at different misorientation angles.

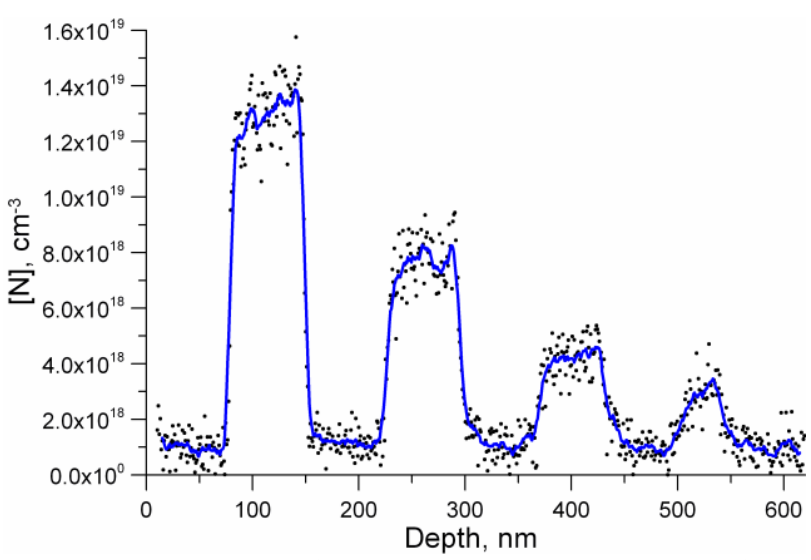

Fig. 1. SIMS profile of the Sample 1. Four nitrogen doped layers was grown at nitrogen flows 1,2, 4 and $8 \mathrm{sccm}$. Methane flow $1.4 \mathrm{sccm}$

Our reactor is capable to vary all of the growth conditions separately during a one growth process. For the temperature variation in a one process the substrate heater was used. The growth was performed under the following conditions: pressure 40 Torr, hydrogen flow $950 \mathrm{sccm}$, methane flow $0.7-1.7 \mathrm{sccm}$. Nitrogen flow was varied from 1 to $8 \mathrm{sccm}$.

Type IIa HPHT diamond (100)-oriented substrates from New Diamond Technologies [3], 3.5 $\times 3.5 \times 0.5 \mathrm{~mm}^{3}$, were used for the nitrogen-doped CVD diamond layers growth. The substrates were pre-treated by ICP plasma in order to get rid of the defects introduced during the polishing procedure [4]. ICP etching up to $5 \mu \mathrm{m}$ depth did not change the surface roughness but allowed to eliminate polishing damages.

Nitrogen incorporation during the process of CVD diamond synthesis was studied at different growth conditions: nitrogen flow, substrate temperature, methane content. High nitrogen concentration $>10^{19} \mathrm{~cm}^{-3}$ was obtained using (100)-oriented HPHT substrates. SIMS was used to measure the nitrogen concentration in the grown diamond samples. The time-of-flight secondary ion mass spectrometer TOF.SIMS-5 by ION-TOF (Münster, Germany) was used for the depth profiling and a Talysurf CCI 2000 interferometer was used to measure the depths of the etching craters for normalization of the analysis depth scale. The instrument operates in dual beam mode employing $1 \mathrm{keV} \mathrm{Cs}+$ ions for sputtering and $25 \mathrm{keV} \mathrm{Bi+}$ ions for probing with $45^{\circ}$ of incidence for both ion beams. The SIMS nitrogen measurements were quantitatively calibrated using a nitrogen ion-implanted HPHT 
single crystal "test" diamond, having the implanted nitrogen concentration equal to $10^{20} \mathrm{~cm}^{-3}$. SIMS profiles were used also for the determination of the growth rate in each of the growth regime.

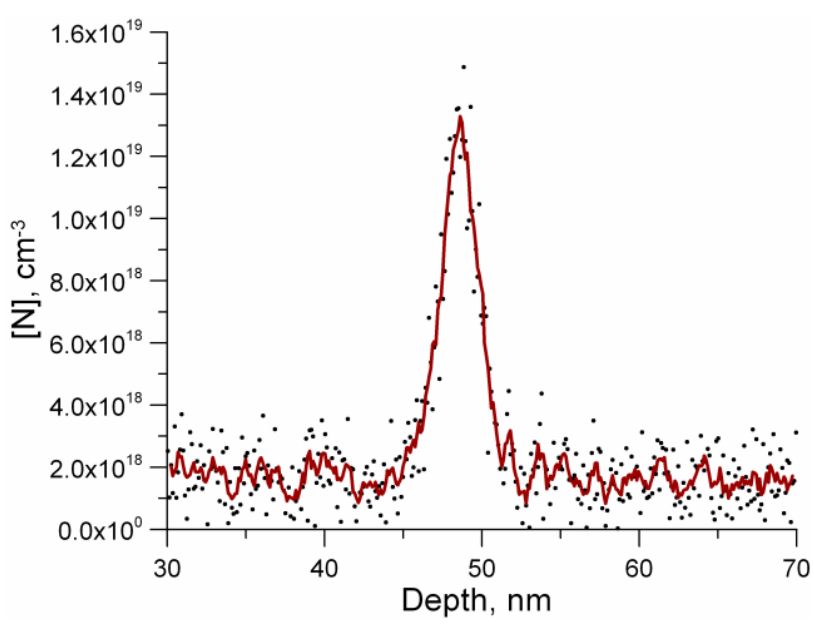

Fig. 2. SIMS profile of nitrogen doped delta-layer (thickness $\sim 3 \mathrm{~nm}$ )

Photoluminescence of the grown samples was measured using micro-Raman system with laser excitation wavelength of $514 \mathrm{~nm}$. Measurements were performed at liquid nitrogen temperature. The results of photoluminescence measurement for the Sample 1 are shown in Fig. 3. They shows the existence of both $\mathrm{NV}^{0}(575 \mathrm{~nm})$ and $\mathrm{NV}^{-}$ $(637 \mathrm{~nm})$ peaks.

In summary, we investigated nitrogen incorporation during CVD diamond growth at different growth conditions. The influence of substrate temperature, methane content and nitrogen flow on the nitrogen incorporation was extensively studied. In order to eliminate the influence of substrate, each of the experiment was performed on one sample, varying the growth conditions in the one growth process. The grown nitrogen doped layers were investigated by SIMS and photoluminescence spectroscopy. Nitrogen incorporation efficiency in our growth regime was determined as $8 \cdot 10^{-6}$.

Moreover, we succeeded in fabrication of ultra-thin nitrogen doped delta layers with peak nitrogen concentration $\sim 10^{19} \mathrm{~cm}^{-3}$ and thickness $\sim 3 \mathrm{~nm}$ (see Fig. 2). The
SIMS profile of such ultra-thin nitrogen doped layer with high peak concentration is demonstrated for the first time. Such ultra-thin nitrogen-doped layers could be used for the creation of high-density NV center ensembles, which are highly desirable for ultra-sensitive magnetometry applications. Due to the absence of lattice damage, as in the case of ion implantation, NV-centers produced by delta-doping are expected to have better spin properties.

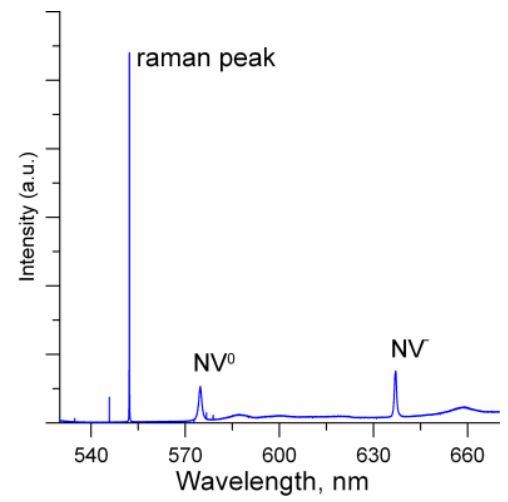

Fig. 3. Photoluminescence spectrum (77 K) of the Sample 1

\section{Acknowledgements}

The study was performed by a grant from the Russian Science Foundation (Project № 16-19-00163).

\section{References}

1. F. Jelezko and J. Wrachtrup, Single defect centers in diamond: a review // Phys. Status Solidi A 2006. V.203. No. 13, P. 3207-3225.

2. A. L. Vikharev, A. M. Gorbachev, M. A. Lobaev, A. B. Muchnikov, D. B. Radishev, V. A. Isaev, V. V. Chernov, S. A. Bogdanov, M. N. Drozdov, and J. E. Butler, Novel microwave plasma-assisted CVD reactor for diamond delta doping // Phys. Status Solidi RRL 2016. V.10. No. 4, P. 324-327.

3. ndtcompany.com

4. A. B. Muchnikov, A. L. Vikharev, J. E. Butler, V. V. Chernov, V. A. Isaev, S. A. Bogdanov, A. I. Okhapkin, P. A. Yunin, and Y. N. Drozdov, Homoepitaxial growth of CVD diamond after ICP pretreatment // Phys. Status Solidi A 2015. V. 212. No. 11, P. 2572-2577. 